\title{
Enhancing Assessment of In-Vehicle Technology Attention Demands with Cardiac Measures
}

\author{
John K. Lenneman \\ Central Michigan University \\ Driver Evaluation, Education \& Research Center \\ Mount Pleasant, MI \\ 517-896-5649 \\ lenne1jk@cmich.edu
}

\author{
Richard W. Backs \\ Central Michigan University Department of Psychology \& \\ Driver Evaluation, Education \& Research Center \\ Mount Pleasant, MI \\ 989-774-6497 \\ backs1rw@cmich.edu
}

\begin{abstract}
In this paper, the differences between driving performance and cardiac measures in attention assessment research are discussed, particularly with regard to evaluating in-vehicle technology design. A number of ways to enhance a set of measures for the purposes of attention assessment are discussed. Finally, the benefits of including cardiac measures as part of a set of IVT attention assessment tools are discussed.
\end{abstract}

\section{General Terms}

Performance, Design, Experimentation, Human Factors.

\section{Keywords}

Psychophysiology, Cardiac, Sensitivity, Diagnosticity.

\section{INTRODUCTION}

As a result of the continuous proliferation of new in-vehicle technologies (IVTs) in the automotive interior, driving is becoming an increasingly complex divided attention task. Research has shown that the increased attention demands imposed by an IVT can have significant detrimental effects on driving performance. [1,2,3] Naturally, the use of driving performance measures in assessing the demands of an IVT on driver attention has become more common, with deteriorations in driving performance viewed as indicators of increased attention demands imposed by side-task IVT performance.

However, the absence of a driving performance decrement does not mean that there are no attention costs that can be attributed to dual-task driving with an IVT task. While it may mean that attention resources are not shared between the two tasks, it may also mean that attention resources were shared but not overtaxed. [4] Relying on performance measures alone to determine the attention demands imposed by an IVT could be insufficient because they might not be sensitive enough to distinguish between these two explanations for the absence of driving performance decrements. Further, the presence of a driving performance decrement does not necessarily provide an indication of which attention resources are affected by IVT task demands (e.g., increased memory load, executive processes). Research has shown that the sensitivity and diagnosticity of a set of measures can be enhanced through the inclusion of cardiac measures across a number of transportation domains, including driving. [5] Sensitivity has been defined as the ability of a measure to detect

Copyright held by author(s)

AutomotiveUI'10, November 11-12, 2010, Pittsburgh, Pennsylvania ACM 978-1-4503-0437-500. changes in task difficulty or resource demand, and diagnosticity as the ability of a measure to indicate the resource, or psychological process, involved in task performance. [6].

\section{ENHANCING SENSITIVITY THROUGH USE OF CARDIAC MEASURES}

Cardiac measures may provide insight into the hidden attentional costs of adding an IVT task to driving that may not manifest in reduced driving performance. For example, some studies have demonstrated an occasional inability of measures of lateral position to support hypotheses that the use of mobile telephones would lead to increases in lateral deviations. [7,8] In our lab, we have repeatedly shown that demands of a side-task can elicit significant cardiac responses with no effect on driving performance. $[9,10,11]$

In Lenneman and Backs [10], subjects were asked to concurrently perform a simulated driving task and a verbal working memory task. While driving, subjects had to maintain their position in the center of the lane and also perform an $n$-back letter recall task (the subject had to decide if the current letter presented matches the $n$th letter previously presented). Compared to driving only, dualtask driving and $n$-back performance elicited a significant response in measures of cardiovascular reactivity, but no change in driving performance. Heart rate increased significantly and high frequency heart rate variability (HF-HRV) decreased significantly, while there was no significant effect on the measure of lateral control. This pattern held as the $n$-back task increased in difficulty (i.e., as the task changed from a 0 -back item recognition task to a 3-back working memory task).

In Lenneman et al. [11], subjects were asked to concurrently perform a simulated driving task and one of two possible sidetasks: one designed to require the processing of focal visual information and another designed to require the processing of ambient visual information. Focal visual information requires attention resources in order to extract the necessary visual features for processing, while ambient visual information does not require attention resources in order to process ego-motion relevant information. [12] Adding the ambient side-task to driving did not have a significant effect on either the lane keeping task or the headway maintenance task. On the other hand, adding the focal side-task to driving did not have an effect on lane keeping, but it did cause a significant deterioration in headway maintenance. However, heart rate increased significantly as a result of adding either side-task to driving. 


\section{ENHANCING DIAGNOSTICITY THROUGH USE OF CARDIAC MEASURES}

In addition to enhancing the sensitivity of a set of measures, the use of cardiac measures can also increase diagnosticity. Past research has demonstrated that certain psychological processes can be mapped to particular cardiac responses. For example, central processing tasks (e.g., mental arithmetic) have been shown to elicit decreases in pre-ejection period (PEP; a measure of sympathetic nervous system influence on the heart) and HF-HRV (or, respiratory sinus arrhythmia, a measure of parasympathetic nervous system influence on the heart). Tasks that elicit executive attentional processes (for higher-order strategies during dual-task performance) elicit only decreases in PEP while tasks that require primarily perceptual/motor processing (e.g., manual tracking) elicit only decreases in HF-HRV. [13,14]

Research has confirmed that these modes of autonomic control are elicited during simulated driving as well. For example, a number of studies have shown that simulated driving in which velocity is fixed (primarily a perceptual/motor processing task) elicits only a decrease in HRV. [9,10] Further, Lenneman \& Backs [10] were able to show that adding a verbal working memory task (primarily a perceptual/central processing task) to simulated driving (primarily a perceptual/motor processing task) elicits a decrease in PEP and HF-HRV. They were also able to demonstrate that cardiac measures can differentiate between performance on the 0 back item recognition task and the 3-back working memory task. Finally, Lenneman et al. [11] were able to show that PEP and HFHRV can differentiate the attention demands of adding focal sidetask to driving from adding an ambient side-task to driving.

\section{APPLICATIONS}

We contend that the use of cardiac measures in driving research may be able to compensate for performance measure insensitivity and a lack of diagnosticity that may exist if only driving performance data are collected when evaluating IVTs. Although developers of an IVT may think they only need to be concerned with whether an IVT actually causes decrements in driving performance, we believe that more insight is needed. For example, developers must consider that that the conditions under which IVTs are tested may change in real-world settings. An IVT that may have had no effect on driving performance in controlled experimental settings may actually cause a deterioration in realworld driving. Because of the enhanced sensitivity and diagnosticity that can be achieved by including cardiac measures, developers may be able to determine which IVT designs exhibit this pattern (i.e., what IVTs are on the precipice of causing a significant deterioration in driving performance). The inclusion of cardiac measures when testing the attention demands of IVT devices can aid developers in designing the least demanding IVTs, and not simply identify those that fail to cause decrements in driving performance in controlled experimental settings.

In summary, cardiac measures can provide sensitive information about the attention demands of IVTs that may not be reflected by driving performance measures, thereby guarding against making incorrect conclusions about the effects of IVT design features on driver workload. Further, adding driver physiology to the set of measures used to evaluate IVTs can help designers within the automotive industry to identify more precisely how the psychological processes necessary for IVT task performance may compete with those necessary for driving.

\section{REFERENCES}

[1] Alm, H., \& Nilsson, L. 1995. The effects of a mobile telephone task on driver behaviour in a car following situation. Accident Analysis and Prevention 27, 707-715.

[2] Brown, I.D., Tickner, A.H., \& Simmons, D.C.V. 1969. Interference between concurrent tasks of telephoning and driving. Journal of Applied Psychology 53, 419-424.

[3] Tsimhoni, O., Smith, D., \& Green, P. 2004. Address entry while driving: Speech recognition versus a touch-screen keyboard. Human Factors 46, 600-610.

[4] Wickens, C.D. 2002. Multiple resources and performance prediction. Theoretical Issues in Ergonomics Sciences, 3, 159-177.

[5] Wilson, G. F. 2002. Psychophysiological test methods and procedures. In S. G. Charlton \& T. G. O'Brien (Eds.), Handbook of human factors testing and evaluation (2nd ed., pp. 127-156). Mahwah, NJ: Erlbaum.

[6] O'Donnell, C. R., \& Eggemeier, F. T. 1986. Workload assessment methodology. In Handbook of perception and human performance: Vol. II. Cognitive processes and performance, K. R. Boff, L. Kaufman, \& J. P. Thomas, Eds. New York: Wiley, 42.1-42.29.

[7] Alm, H., \& Nilsson, L. 1995. The effects of a mobile telephone task on driver behaviour in a car following situation. Accident Analysis and Prevention, 27, 707-715.

[8] Parkes, A.M. \& Hooijmeijer, V. 2001. Driver situation awareness and carphone use. In Proceedings of the $1^{\text {st }}$ Human-Centered Transportation Simulation Conference (n.p.). Iowa City: University of Iowa.

[9] Lenneman, J.K., Shelley, J.R., \& Backs, R.W. 2005. Deciphering psychological-physiological mappings during a simulated driving task. In Proceedings of the Third International Driving Symposium on Human Factors in Driver Assessment, Training, and Vehicle Design. Rockland: ME, 493-498

[10] Lenneman, J.K., \& Backs, R.W. 2009. Cardiac autonomic control during simulated driving and a concurrent verbal working memory task. Human Factors 51, 404-418.

[11] Lenneman, J.K., Cassavaugh, N., \& Backs, R.W. in preparation. A psychophysiological and driving performance evaluations of visual channel demand while driving.

[12] Previc, F.H. 1998. The neuropsychology of 3-D space. Psychological Bulletin 124, 123-164.

[13] Backs, R.W., Rhody, J., \& Barnard, J. 2005. Cardiac control during dual-task performance of visual or auditory monitoring with visual-manual tracking. Psychologia 48, 6683.

[14] Berntson, G.G., Cacioppo, J.T., \& Fieldstone, A. 1996. Illusions, arithmetic, and bi-directional modulation of vagal control of the heart. Biological Psychology 44, 1-17. 